\title{
Management Method for Large Wheel Loads and Lateral Forces Using Axle-box Acceleration and Track Irregularity
}

Hirofumi TANAKA

Researcher,
Atsushi FURUKAWA, Dr. Eng.

Senior Researcher, Laboratory Head,

Track Geometry and Maintenance Group, Track Technology Division

Very large wheel loads and lateral forces are frequently caused by short-wavelength track irregularities. These large loads are undesirable, as they have a significant effect on the deterioration of the track material and necessitate additional track maintenance. This study focused on correlation between wheel load, lateral force, axle-box acceleration and track irregularity. First, we analyzed the frequency characteristics of the relationship between wheel loads, lateral forces, axle-box acceleration and track irregularities. Then, we proposed a technique to estimate wheel loads and lateral forces that involves a shortwavelength component using axle-box acceleration and track irregularity waveforms.

Keywords: vertical axle-box acceleration, lateral axle-box acceleration, large wheel load, large lateral force, frequency response method, short-wavelength track irregularity

\section{Introduction}

Large wheel loads and rolling noise generated by roughness (such as welds or joint depressions) and corrugation on the top surface of rails are causes for concern in the railway industry, in addition to the large lateral forces generated by angular bends at joints. In Japan, axle-box acceleration has been used to cope with these issues for a long time (as referred to in [1, 2, 3 and 4]). As these problems are caused by very short-wavelength track irregularities, it is difficult to comprehend them with the $10-\mathrm{m}$ versine chord measured by the track geometry cars normally used for track maintenance. Axle-box acceleration is the acceleration measured with an accelerometer fixed to the axle-box that supports the wheel set of a vehicle as shown in Fig. 1. In this paper, acceleration in the vertical direction called vertical axlebox acceleration, also horizontal acceleration called lateral axle-box acceleration.

The relationship between large values of wheel load, lateral force and axle-box acceleration has been investigated previously because evaluation of track material durability involves the management of such influences, and an intermittent measuring system has mainly been used to ascertain the wheel load and lateral force values [5]. Here, if the amplitude ratio of axle-box acceleration to the wheel load or the lateral force were constant regardless of the frequency, high-accuracy management would be possible using the above-mentioned system. However, estimation accuracy actually decreases according to the irregularities of the rail surface or rail joint because the amplitude ratio is not constant. Moreover, in recent years, wheel loads and lateral forces have often been checked using the new continuous measuring system when the train operation starts in newly constructed railway lines, or the service operation of newly developed vehicle models starts [6]. The new continuous measuring system is a Japanese technique that measuring wheel loads and lateral forces up to $100 \mathrm{~Hz}$ on running vehicles. Wheel loads, lateral forces and axlebox acceleration can be evaluate using data obtained by this method.

Against this background, we first analyzed the relationship between wheel loads and lateral forces using the new continuous measuring system, and axle-box acceleration was measured at the same time in the frequency domain. In addition, we examined a technique for estimating wheel load and lateral force variations caused by the short-wavelength track irregularity from 1 to $6 \mathrm{~m}$ using axle-box acceleration. We also estimated wheel load and lateral force variations caused by the comparatively long wavelengths track irregularity from six to several tens of meters generally obtained by the 10-m versine chord method using a track geometry car [7]. In addition, we developed a new method to estimate variations in wheel loads and lateral forces. The technique can estimate variations that contain short-wavelength fluctuations (in contrast to the existing technique that uses only track irregularities) by overlapping two estimated waveforms with a different wavelength band.

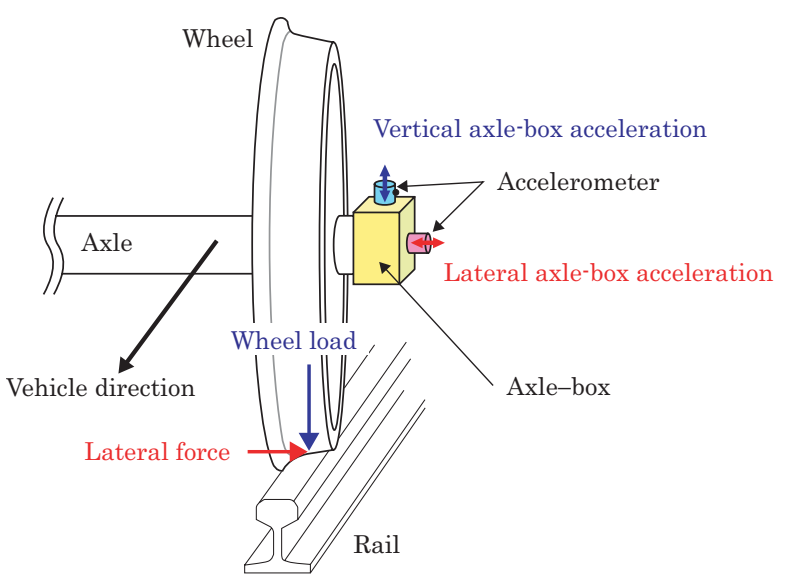

Fig. 1 Outline of wheel load, lateral force and axle-box acceleration 
Moreover, we verified the validity and accuracy of the past technique for estimating large wheel loads and lateral forces that uses large values of axle-box acceleration $[2,4]$. Finally, we proposed a technique to manage large wheel loads and large lateral forces.

\section{Relationship between variations in wheel load, lateral} force, axle-box acceleration and track irregularities

\subsection{Correlation}

Figure 2 shows the correlation between wheel load variation and vertical axle-box acceleration measured on a express vehicle for conventional-line, along with longitudinal level and cross level in the same section. The section examined was a tangent track, and the running speed of the vehicle was $110 \mathrm{~km} / \mathrm{h}$. At spatial frequen-

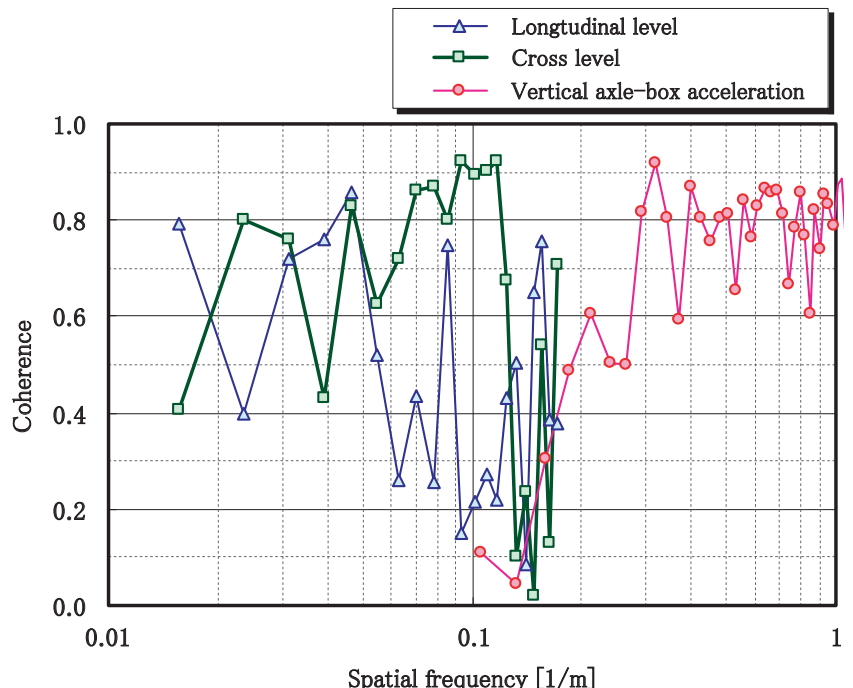

Fig. 2 Correlation between wheel load variation/vertical axle-box acceleration and irregularities in the cross level and longitudinal level (tangent section, 110 $\mathrm{km} / \mathrm{h}$ )

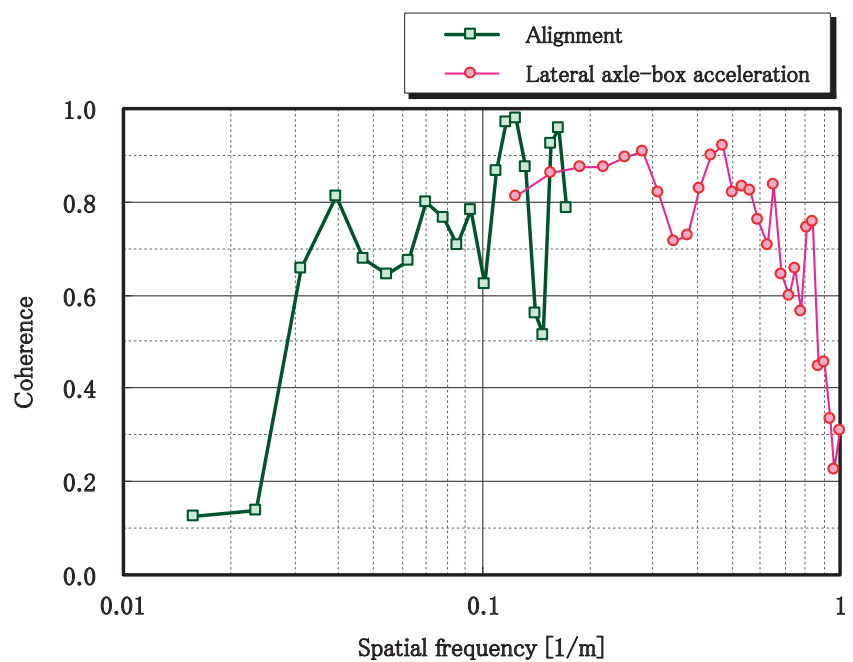

Fig. 3 Correlation between lateral force variation/lateral axle-box acceleration and irregularities of alignment (curved section, $85 \mathrm{~km} / \mathrm{h}$ ) cies lower than $0.1[1 / \mathrm{m}]$, the wheel load correlates to the cross level, while in the frequency band from 0.03 to 0.05 $[1 / \mathrm{m}]$ it correlates to the longitudinal level. At spatial frequencies higher than $0.167[1 / \mathrm{m}]$, the wheel load correlates to the vertical axle-box acceleration. As the power of the wheel load variation is originally small from 0.15 to $0.25[1 / \mathrm{m}]$, the correlation between wheel load, track irregularity and vertical axle-box acceleration is low.

Figure 3 shows the correlation between lateral force variation and lateral axle-box acceleration measured on a express vehicle for conventional-line and alignment in the same section. The section examined was a curved track, and the running speed of the vehicle was $85 \mathrm{~km} /$ $\mathrm{h}$. At spatial frequencies lower than $0.167[1 / \mathrm{m}]$, the lateral force correlates to the alignment, while at frequencies higher than $0.1[1 / \mathrm{m}]$ it correlates to the lateral axle-box acceleration.

Hence, wheel load and lateral force variations with a wavelength of less than $6 \mathrm{~m}$ (which cannot be clarified by track irregularity waveforms using the 10-m chord versine method) can be evaluated from axle-box acceleration.

\subsection{Frequency properties}

Figure 4 shows the frequency response functions between wheel load, vertical axle-box acceleration, longitudinal level and cross level in the same section as that in Fig. 2. The longitudinal level shows restoration waveform with a wavelength higher than $6 \mathrm{~m}$. The amplitude gain of wheel load variation for the cross level and longitudinal level grows with increased spatial frequency. A smooth amplitude gain is shown for spatial frequencies higher than $0.167[1 / \mathrm{m}]$ in terms of the relationship between vertical axle-box acceleration and wheel load variation.

Figure 5 shows the frequency response functions between lateral force, lateral axle-box acceleration and alignment in the same section as that in Fig. 3. The alignment shows restoration waveform with a wavelength

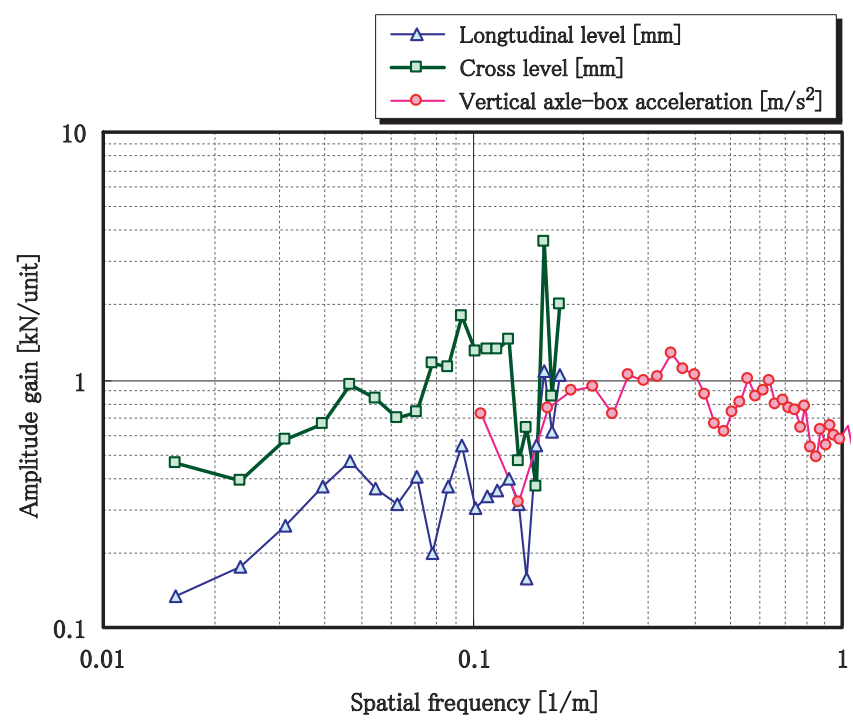

Fig. 4 Frequency response function of wheel load variation (tangent section, $110 \mathrm{~km} / \mathrm{h}$ ) 


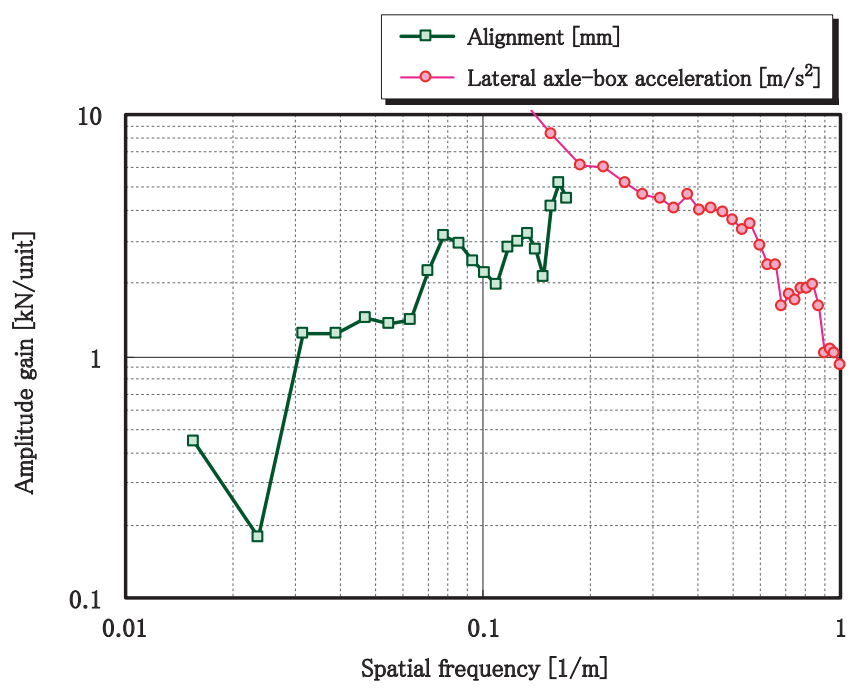

Fig. 5 Frequency response function of lateral force variation (curved section, $85 \mathrm{~km} / \mathrm{h}$ )

higher than $6 \mathrm{~m}$. The amplitude gain of lateral force variation by alignment grows with increased spatial frequency. The amplitude gain becomes small for the relationship between lateral axle-box acceleration and lateral force variation with increased spatial frequency. Wheel load variation is therefore strongly influenced by short-wavelength track irregularities with a wavelength frequency of less than $6 \mathrm{~m}$.

\section{Estimation technique based on the frequency response method}

\subsection{Details of estimation technique}

This chapter examines the technique for estimating wheel load and lateral force variation using the frequency response method [8], which estimates output waveforms from input waveforms using a FIR filter. This filter uses an impulse response (the reverse-Fourier transform of the frequency response function of the I/O waveform) based on the fast Fourier transform. Examination has previously been based on this technique, but only variations with a wavelength higher than $6 \mathrm{~m}$ could be estimated because the input waveform consists only of track irregularity [7]. Accordingly, axle-box acceleration in addition to those of track irregularities were used as input waveforms in this method.

Figure 6 shows an outline of the technique for estimating wheel load and lateral force variation based on axle-box acceleration together with track irregularities. These irregularities are adopted to estimate the variations with wavelengths longer than $6 \mathrm{~m}$ as usual. The input waveforms of track irregularities have a high correlation with wheel load and lateral force variation. The cross level is used to estimate wheel load variation, and the alignment is used to estimate lateral force variation. As an input waveform, estimation of variation for wavelengths of less than $6 \mathrm{~m}$ is based on vertical axle-box acceleration for wheel load variation and lateral axlebox acceleration for lateral force variation. In addition,

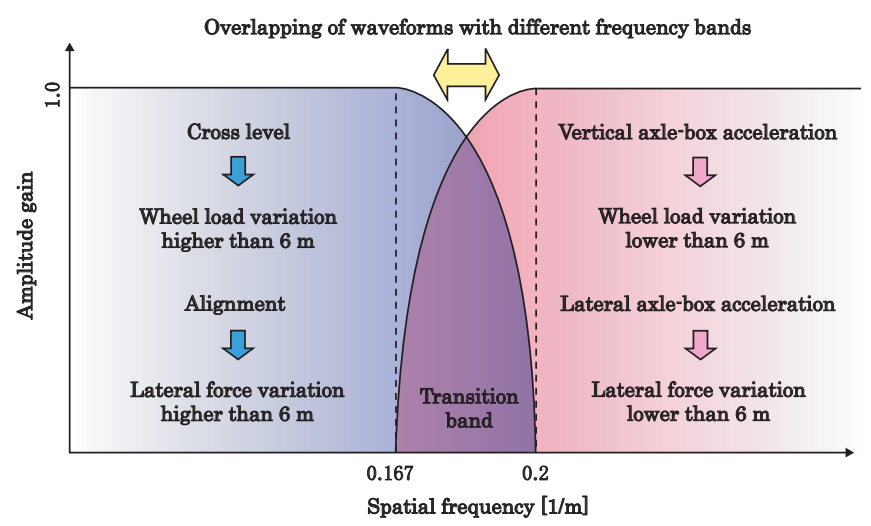

Fig. 6 Outline of estimation method for wheel load and lateral force variation using axle-box acceleration with track irregularities

two estimated waveforms with different wavelengths are overlapped in both the cases of wheel load and lateral force variation. Thus, the final estimated waveform contains the variation of the short-wavelength from the previous estimation technique. In this study, it is necessary to install a transition band in the cutoff frequency as a characteristic of the digital filter. In this technique, wavelengths of 5 to $6 \mathrm{~m}$ (spatial frequency 0.167 to 0.2 $[1 / \mathrm{m}]$ ) are considered as the transition band so that amplitude gain after overlapping becomes one at the transition band.

The track irregularity data used here is based on distance sampling data usually obtained at equal intervals of $0.25 \mathrm{~m}$ by the track geometry car. However, axlebox acceleration was first measured on the vehicle with a distance pulse by time sampling at several thousand $\mathrm{Hz}$. The results were then converted into data for distance sampling of several centimeters through off-line processing. Therefore, since the sampling interval of both waveforms is different, it is necessary to match the sampling interval while nothing any aliasing.

\subsection{Estimated results of wheel load and lateral force variation}

Figure 7 shows the good agreement between measured and estimated wheel load waveforms. The estimation track was a straight section different from the one where the frequency response was obtained, and the running speed of the vehicle was $110 \mathrm{~km} / \mathrm{h}$. The cross level and the waveform estimated from it were processed using a band-pass filter (BPF) set to a wavelength band of $6-25 \mathrm{~m}$, and the vertical axle-box acceleration and the waveform estimated from it were treated using a BPF with a wavelength band of $1-6 \mathrm{~m}$. A smooth wheel load variation with long wavelength was estimated from the cross level. An impact wheel-load variation exited by local vertical track irregularity at rail joints with $25 \mathrm{~m}$ intervals is shown clearly by the waveform estimated from the vertical axle-box acceleration. In addition, the final estimated waveform that overlaps both estimated waveforms corresponds satisfactorily to the measured wheel load variation.

Figure 8 also shows the good agreement between the 
measured and estimated lateral force waveforms of the outer rail. The estimation track was a curved section of $400 \mathrm{~m}$ in radius (different from the section where the frequency response was obtained), and the running speed of the vehicle was $85 \mathrm{~km} / \mathrm{h}$. The alignment and the waveform estimated from it were processed with a BPF set to a wavelength band of $6-25 \mathrm{~m}$, and the lateral axle-box acceleration and the waveform estimated from it were treated using a BPF with a wavelength band of $1-6 \mathrm{~m}$. Lateral force variation with long wavelength was esti- mated from the alignment. An impact lateral force variation excited by local lateral track irregularity at rail joints with $25 \mathrm{~m}$ intervals is clearly shown by the waveform estimated from the lateral axle-box acceleration. In addition, the final estimated waveform that overlaps both estimated waveforms corresponds satisfactorily to the measured lateral force variation.

Figure 9 shows the comparison of large values of wheel load variation estimated from the vertical axlebox acceleration and the measured wheel load variation.

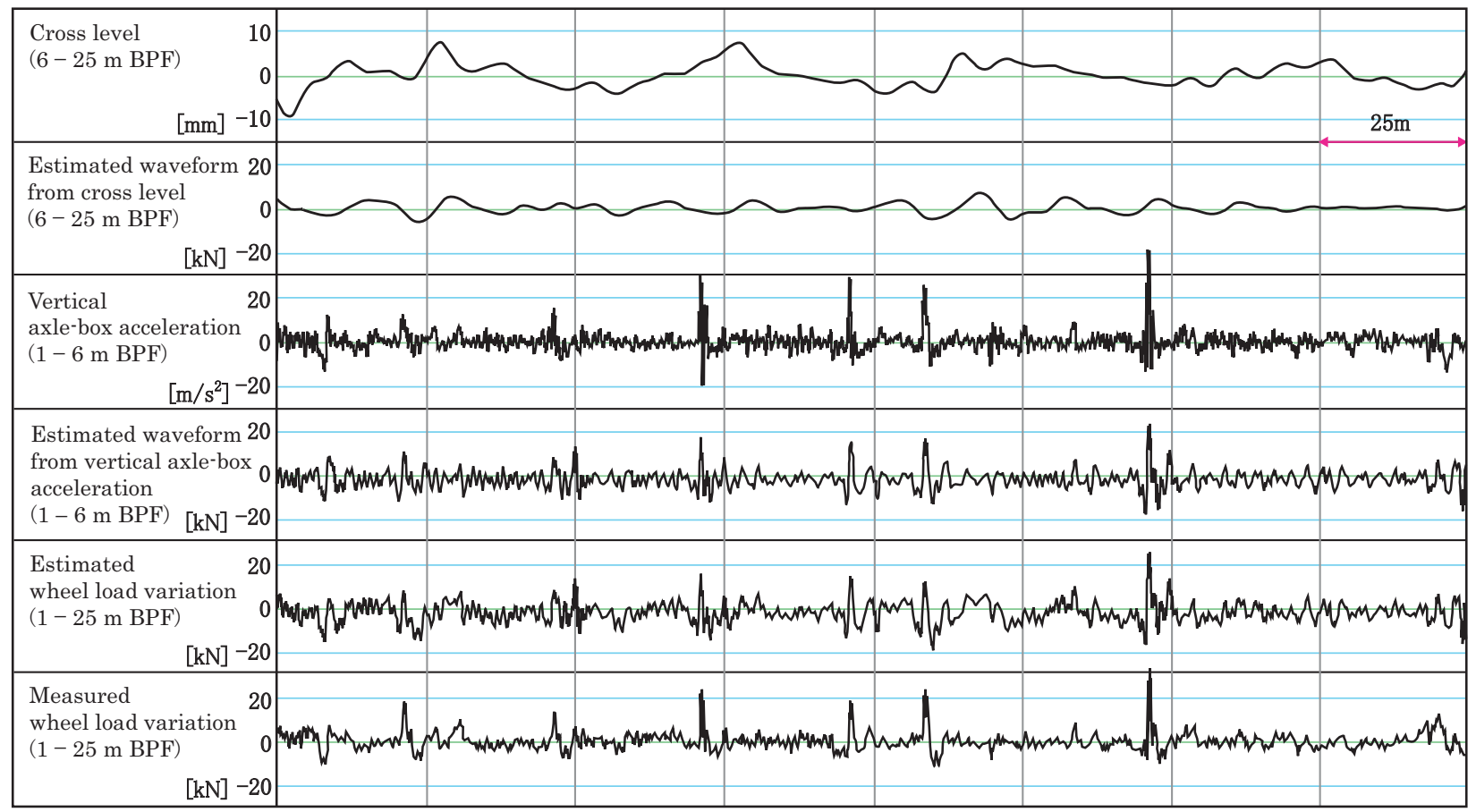

Fig. 7 Measured and estimated waveforms of wheel load variation (tangent section, $110 \mathrm{~km} / \mathrm{h}$ )

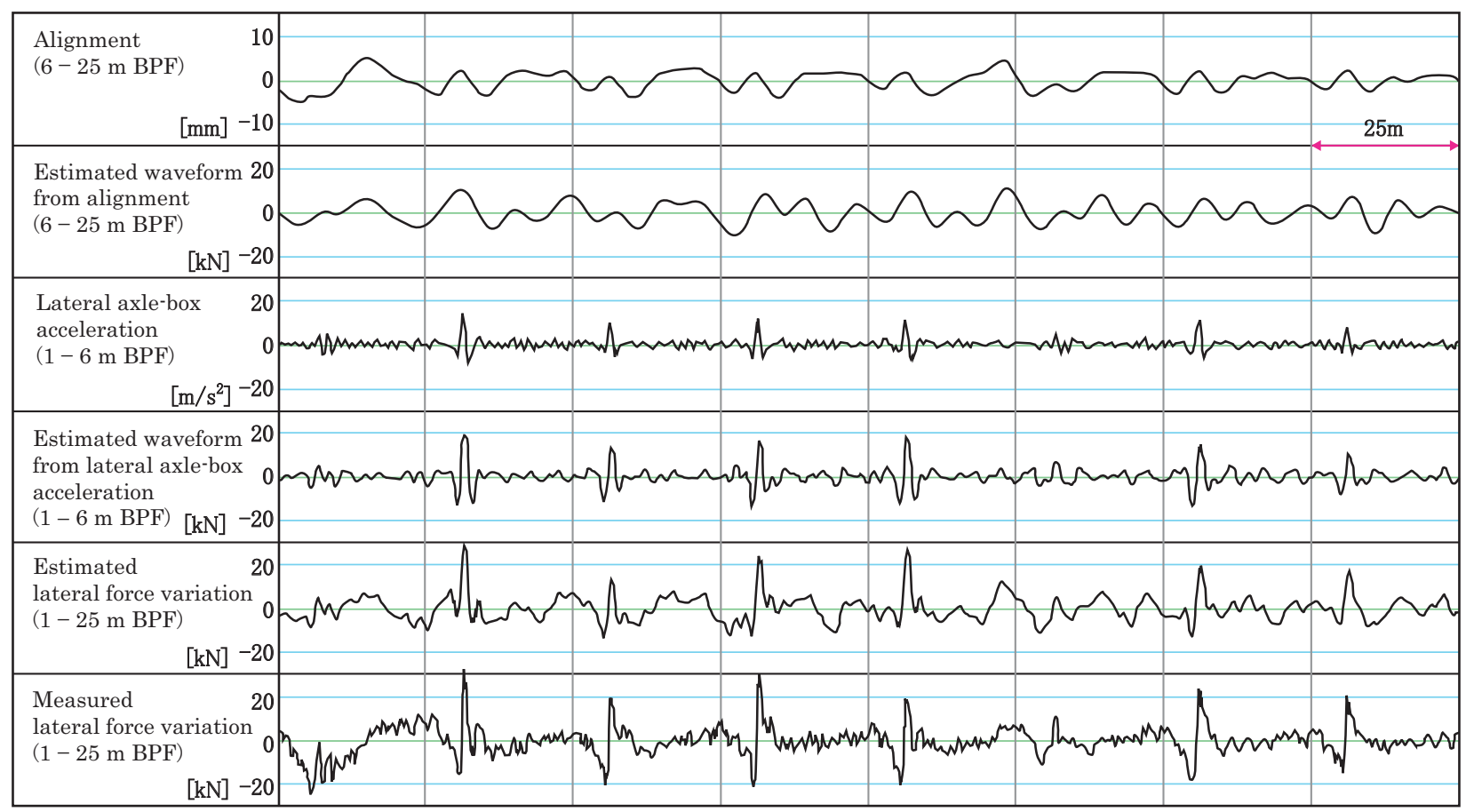

Fig. 8 Measured and estimated variation of lateral force waveforms (curved section, $85 \mathrm{~km} / \mathrm{h}$ ) 


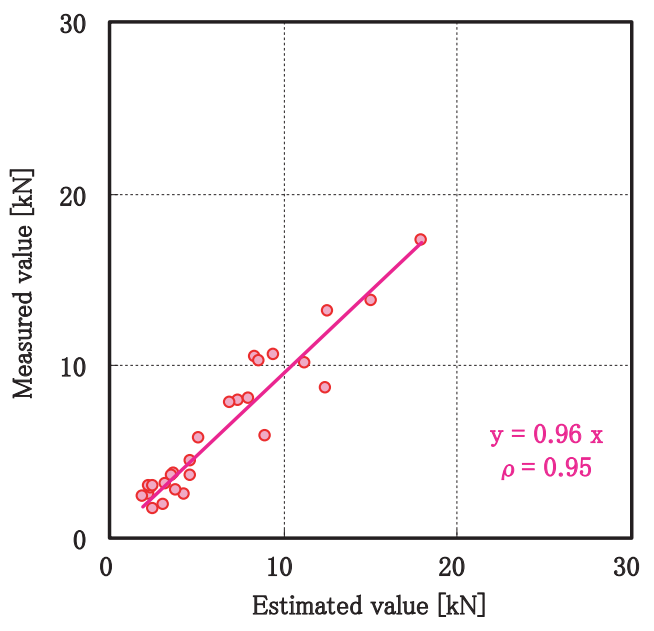

Fig. 9 Large values of measured and estimated waveforms for wheel load variation (frequency band $=1-6 \mathrm{~m}$ )

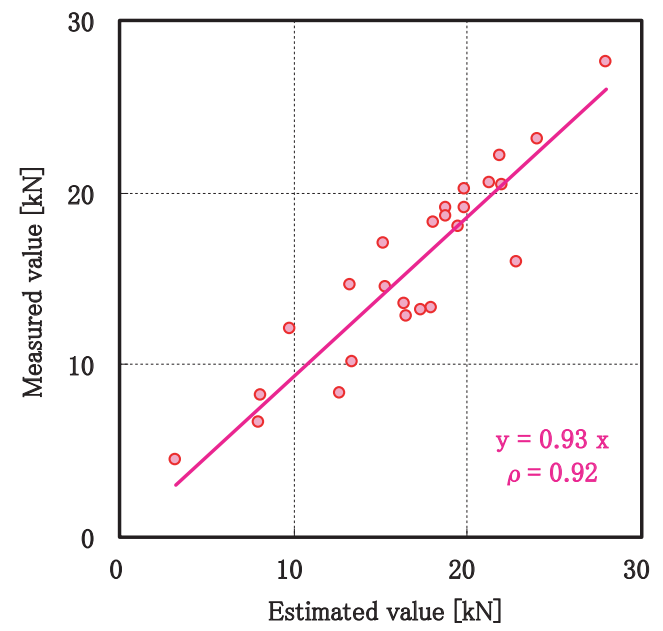

Fig. 10 Large values of measured and estimated waveforms for lateral force variation (frequency band $=1-6 \mathrm{~m}$ )

The frequency component of both waveforms consists of wavelengths of less than $6 \mathrm{~m}$. Relatively large values forced on in this study were often found at rail joints with $25 \mathrm{~m}$ intervals, and were calculated with a maximum value of $25 \mathrm{~m}$. The correlation between large values of estimated waveform for the wheel load variation and the measured waveform is very high.

Figure 10 shows the comparison of large values of lateral force variation estimated from the lateral axlebox acceleration and the measured lateral force variation, both treated with a BPF of less than $6 \mathrm{~m}$. In the same way as above, the large values were calculated with a maximum value of $25 \mathrm{~m}$. The correlation between large values of estimated waveform for the lateral force variation and the measured waveform is very high. This demonstrates that the technique is useful not only for estimating wheel load and lateral force variations of shortwavelength but also for estimating these large values.
4. Estimation of large wheel loads and lateral forces from large values of axle-box acceleration

\subsection{Technological significance of estimating large values using a simple method}

We demonstrated that wheel load and lateral force variation with short-wavelengths could be estimated with high accuracy using the frequency response method, which involves inputs of waveform axle-box acceleration and track irregularities. However, a processing environment and knowledge of time series data are needed to enable these processes. The amplitude of impact wheel load and lateral force variation with short-wavelength is generally larger than gradual wheel load and lateral force variation with long wavelength. Since the absolute values of wheel load and lateral force variation estimated from axlebox acceleration are larger than those of wheel load and lateral force variations estimated from track irregularities in the estimation waveforms as shown in Fig. 7 and Fig. 8.

Conversely, a simple technique for estimating large wheel loads and large lateral forces has been developed using large values of axle-box acceleration as described in the first chapter [2], [4]. Since the waveforms of wheel load, lateral force and axle-box acceleration are similar (as shown in Figs. 7 and 8), large values of both are approximated by the expression, and large wheel loads or lateral forces are estimated from large values of axle-box acceleration. This technique is referred to here as the simple method. If the amplitude gain of the frequency response function for wheel load and lateral force variation to axle-box acceleration were constant regardless of spetial frequencies, this method would be correct. However, the amplitude gain of an actual frequency response function is not necessarily completely constant (as shown in Figs. 4 and 5) compared with frequency, making it necessary to confirm the validity of the simple method.

In this chapter, we compare the estimated results for large wheel loads and lateral forces estimated using the frequency response method with those calculated using the simple method. We also verify the estimation accuracy for large values from both techniques and examine the scope of application for the simple method.

\subsection{Comparison of large values}

In the frequency response function of wheel load variation and vertical axle-box acceleration shown in Fig. 4 , the amplitude gain was smooth. This suggests that large values of wheel load and vertical axle-box acceleration can be approximated by a linear equation, and that large wheel loads can be estimated by large values of vertical axle-box acceleration. Figure 11 shows a comparison of large values for wheel loads and vertical axlebox acceleration. The section examined was the same as that in Fig. 9. Each large value was calculated with a maximum value of $25 \mathrm{~m}$. To extract only impact variations, both waveforms were processed using a BPF with a wavelength $1-6 \mathrm{~m}$. Figure 11 shows that both sets of large values demonstrate a high correlation. The inclination of the regression is 0.77 , and is almost equal to 
the mean value of amplitude gain for the frequency band of the frequency response function for wavelengths of 1 $6 \mathrm{~m}$ shown in Fig. 4.

On the other hand, in the frequency response function of lateral force variation and lateral axle-box acceleration shown in Fig. 5, amplitude gain falls as spatial frequency increases. Figure 12 shows the comparison of large values for lateral force and lateral axle-box acceleration. The section examined was the same as that in Fig. 10. In the same way as above, each large value was calculated with a maximum value of $25 \mathrm{~m}$, and both waveforms were processed using a BPF with a wavelength $1-6 \mathrm{~m}$. Figure 12 shows that both sets of large values demonstrate a high correlation that varies slightly compared with the case for wheel load. The inclination of the regression is 3.52 , and is almost equal to the mean value of amplitude gain for the frequency band of the frequency response function for wavelengths of $1-6 \mathrm{~m}$ shown in Fig. 5. As with the case of wheel load, if only the impact of large values is considered, it is possible to approximate large values of lateral force and lateral axle-

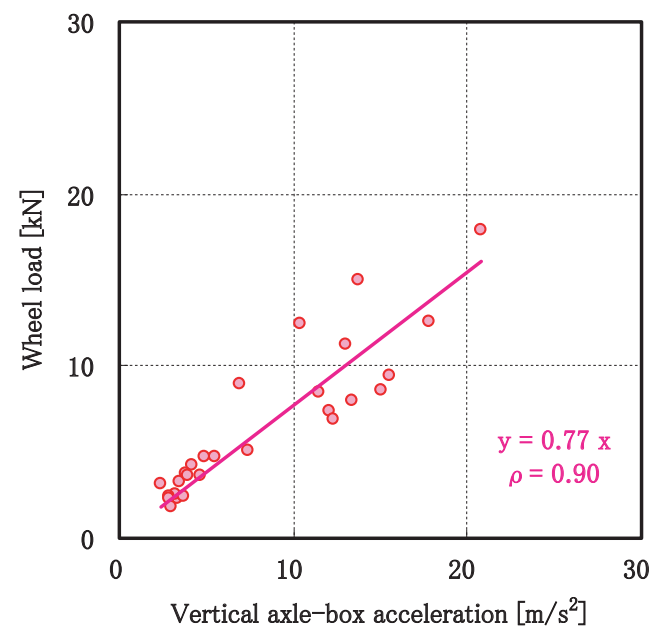

Fig. 11 Relationship between large values of wheel load variation and vertical axle-box acceleration (frequency band $=1-6 \mathrm{~m}$ )

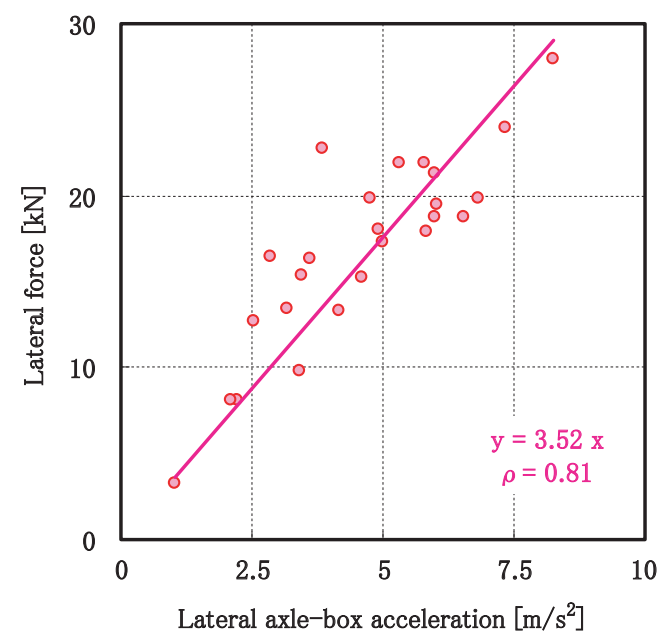

Fig. 12 Relationship between large values of lateral force variation and lateral axle-box acceleration (frequency band = $1-6 \mathrm{~m}$ ) box acceleration with a linear equation, even though the amplitude gain is not smooth [4].

As a result, it is not as small as it becomes a problem for practical use even though the correlation coefficient obtained using the simple method is lower than that obtained using the frequency response method. In particular, because the amplitude gain of the frequency response function is smooth and the amplitude gain almost corresponds to the regression coefficient of the simple method, large wheel loads can be estimated with sufficient accuracy using the simple method.

\section{Management method for large wheel loads and lateral force}

Wheel load variation and lateral force variation with short-wavelength can be estimated from axle-box acceleration. We have also shown that it is possible to identify where large wheel loads and large lateral forces will be generated, as well as their extent. However, for efficient track maintenance, it is necessary to understand track irregularities of short-wavelength that cause the generation of large wheel loads and large lateral forces. Track irregularity measurement using the asymmetrical chord offset method performed by a two-bogie-type track geometry car is suitable for this purpose [9]. This method can detect track irregularities with wavelengths shorter than $6 \mathrm{~m}$ as it differs from the 10 -m chord versine method, but it is difficult to maintain an actual track with an asymmetrical chord offset. It is easier to deal with track irregularity waveforms when they are converted into the versine waveform of a short chord. In this regard, it is appropriate to convert the asymmetrical chord offset into a versine waveform chord of 4 or 5 $m$ in consideration of conversion accuracy.

Figure 13 shows a conceptual diagram of the management procedure for large wheel loads and lateral forces using axle-box acceleration values and track irregularity waveforms with an asymmetrical chord offset. First of all, the points at which large wheel loads and large lateral forces occur are extracted using the method outlined in Chapter 3. As a result of this estimation, short-wavelength track irregularities are expected to arise at points estimated to generate large values that exceed the reference value. In this case, it is necessary to set the management value in consideration of differences in the estimated results. The maintenance of short-wavelength track irregularities uses the waveforms of asymmetrical chord offsets measured with a twobogie-type track geometry car. Using the asymmetrical chord offset waveform enables appropriate management and maintenance of track irregularities that represent generation points of large wheel loads and lateral forces with regular track measurement.

Large wheel loads and lateral forces generated by short-wavelength track irregularities change greatly depending on the running speed of the vehicle. It is therefore undesirable to set a reference value for the magnitude of track irregularities (as has been done in past track maintenance) and manage it. As a management standard, it is appropriate to maintain large wheel 


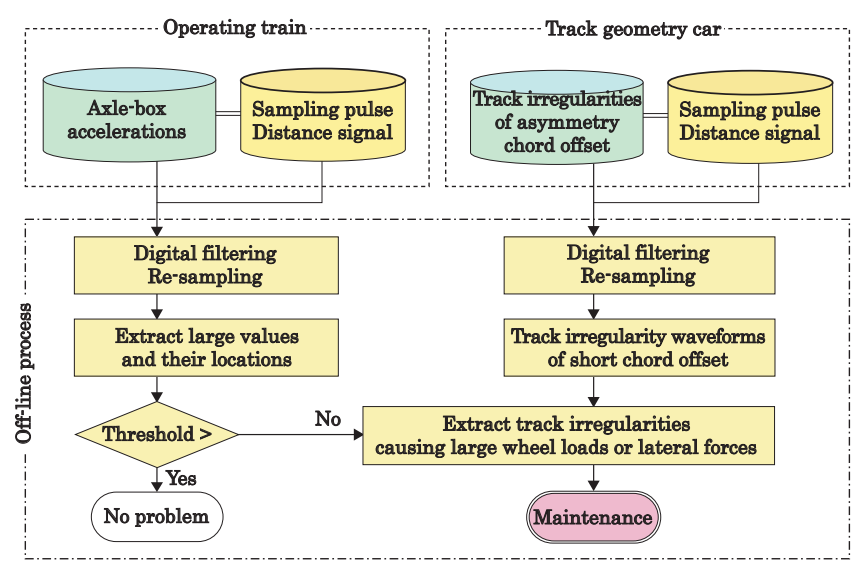

Fig. 13 Flowchart of management procedure for large wheel loads and lateral forces

loads and lateral forces decided from the durability of track material from the viewpoint of load managing loads that act on the track. Finally, the parameters used in this paper (such as the impulse response used in the frequency response method or the coefficients used in the simple method) apply only to the vehicle type examined in this study. It is important to examine the relationships between wheel loads, lateral forces and axle-box acceleration, for example, for a typical vehicle running in a maim line to enable the application of the management technique described here to commercial environments.

\section{Conclusion}

In this paper, we analyzed the relationships between wheel loads, lateral forces, axle-box acceleration and track irregularities, and obtained the following results:

(1) An estimation technique for wheel load and lateral force variation was developed based on the frequency response method (involving input waveforms of track irregularity and axle-box acceleration) and estimated wheel load and lateral force variation. As a result, we were able to calculate wheel load and lateral force variations, as well as large wheel loads and lateral forces consisted of a frequency component with a wavelength of less than $6 \mathrm{~m}$ that could not be estimated using the past technique in which only track irregularities are input.

(2) We verified the validity of a simple method to esti- mate large values of wheel load and lateral force from large values of axle-box acceleration. As a result, we showed that large wheel loads and large lateral forces could be estimated without complex signal conditioning from large values of axle-box acceleration.

(3) Finally, we demonstrated a management procedure using track irregularities that converts asymmetrical chord offsets into short versine chord offsets, and a way of maintaining track irregularities of shortwavelength in which large values arise.

\section{References}

[1] Sunaga, Y., Ide, T. and Kanao, M., "A Practical Use of Axle-box Acceleration to Control the Short-wave Track Irregularities on Shinkansen," RTRI Report, Vol. 9, No. 2, pp. 35 - 40, 1995 (in Japanese).

[2] Sunaga, Y., Sano, I. and Ide, T., "A Method to Control the Short Wave Track Irregularities Utilizing Axlebox Acceleration," QR of RTRI, Vol. 38, No. 4, pp. $176-181,1997$.

[3] Yoshida, M, Shinowaki, S. and Sunaga, Y., "A Study on Lateral Axle-box Acceleration for Detecting Irregularities of Rail Joints on Sharp Curves," Jointed Railway Technology Symposium (J-Rail2004), Vol. 11, pp. 91 - 94, 2004 (in Japanese).

[4] Tanaka H. and Furukawa A., "The estimation method of wheel load and lateral force using the axle-box acceleration," presented at the $W C R R$, Seoul, Korea, May 19 - 22, 2008.

[5] Hayashi S. and Miyaishi G., "Automatic data processor of wheel load and lateral load of rolling stock," Railway Technical Research Report, No. 1008, p. 29, 1976 (in Japanese).

[6] Ishida, H., Ueki, K., Fukazawa, K., Tezuka, K. and Matsuo, M., "A New Continuous Measuring Method of Wheel/Rail Contact Forces," QR of RTRI, Vol. 35, No. 2, pp. 105 - 111, 1994.

[7] Furukawa A., "A Method to Predict Vehicle Wheel Load Induced by Track Irregularity," RTRI Report, Vol. 19, No. 2, pp. 35 - 40, 2005 (in Japanese).

[8] Julius S. Bendat and Allan G. Piersol, Random Data - Analysis and Measurement Procedures, John Wiley \& Sons, Inc., USA, p. 396, 1971.

[9] Takeshita, K., "A Method for Track Irregularity Inspection by Asymmetrical Chord Offset Method," QR of RTRI, Vol. 33, No. 2, pp. 106 - 114, 1992. 